\title{
Electrostatic Mechanism for Depolymerization-Based Poleward Force Generation at Kinetochores
}

\author{
L. John Gagliardi' ${ }^{1}$, Daniel H. Shain ${ }^{2}$ \\ ${ }^{1}$ Department of Physics, Rutgers The State University of New Jersey, Camden, NJ, USA \\ ${ }^{2}$ Department of Biology, Rutgers The State University of New Jersey, Camden, NJ, USA. \\ Email:gagliard@scarletmail.rutgers.edu,dshain@camden.rutgers.edu
}

How to cite this paper: Gagliardi, L.J. and Shain, D.H. (2019) Electrostatic Mechanism for Depolymerization-Based Poleward Force Generation at Kinetochores. Open Journal of Biophysics, 9, 198-203. https://doi.org/10.4236/ojbiphy.2019.93014

Received: June 1, 2019

Accepted: July 22, 2019

Published: July 25, 2019

Copyright $\odot 2019$ by author(s) and Scientific Research Publishing Inc. This work is licensed under the Creative Commons Attribution International License (CC BY 4.0).

http://creativecommons.org/licenses/by/4.0/

\begin{abstract}
Experiments implicating bound volume positive charge at kinetochores interacting with negative charge at microtubule free ends have prompted our calculation of the force at kinetochores for chromosome poleward motility during mitosis. We present here a corroborating force calculation between positively charged $\mathrm{Hec} 1$ tails in kinetochores and negatively charged C-termini at microtubule free ends. Based on experimentally-known charge magnitudes on $\mathrm{Hecl}$ tails and C-termini at microtubule free ends, an $a b$ initio calculation of poleward (tension) force per microtubule that falls within the experimental range is demonstrated. Due to the locations of C-termini charges on concave sides of splaying microtubules, this attractive force between subsets of low curvature splaying microtubule protofilaments C-termini eventually fails for subsets of protofilaments with more pronounced curvature, thus generating poleward force as microtubules depolymerize in a dynamic coupling, as observed experimentally. The mechanism by which kinetochores establish and maintain a dynamic coupling to microtubules for force production during the complex motions of mitosis remains elusive, and force generation at kinetochores has emerged as a signature problem in chromosome motility. In agreement with experiment, two separate calculations show that attractive electrostatic interactions over nanometer distances account for poleward chromosome forces at kinetochores.
\end{abstract}

\section{Keywords}

Mitosis, Chromosome, Motility, Force, Electrostatics

\section{Background}

Force generation at kinetochores has emerged as one of the signature problems 
in mitotic movements. Consistent with theoretical predictions made over a decade ago [1] [2], electrostatic interactions at kinetochores between negatively charged microtubule plus ends and positive charge at kinetochores have more recently been proposed for chromosome motility during mitosis [3]. A number of currently advanced models involve interactions that are fundamentally electrostatic, including mechanisms for chromosome movements based on protofilament-end splaying. A brief review of current models for force production at kinetochores is given elsewhere [4], where we support the experimental work of Miller et al. [3] with an ab initio calculation of the force between bound volume positive charge distributions at kinetochores interacting electrostatically with bound negative charge at free ends of microtubules.

Our purpose here is to mathematically corroborate that calculation with one that is based on direct interactions between positively charged unstructured $\mathrm{Ndc} 80 \mathrm{Hec} 1$ tails in kinetochores and negatively charged C-termini at the free ends of microtubules, supporting an electrostatic-based model that explains poleward force generation.

Miller et al. [3], advances $\mathrm{Ndc} 80 / \mathrm{Hec1}$ as responsible for electrostatics-based force production at kinetochores. They propose that the force-producing interaction is electrostatic since an unstructured positively charged Hec1 tail cannot bind microtubules lacking negatively charged C-termini, concluding that “... the highest affinity interactions between kinetochores and microtubules are ionic attractions between two unstructured domains". Our approach supports the role of $\mathrm{Hecl}$ as bound volume charge distributions-"positively charged Hecl tails" [3] -at kinetochores, interacting electrostatically with bound negative charge at and near the free ends of microtubules- "ionic attractions between two unstructured domains" [3].

Chromosomes can move toward a proximal pole only when their kinetochores are connected to microtubules coming from that pole [5]. Microtubule polymerization and depolymerization follow a pattern characterized as "dynamic instability." This means that at any given time, some of the microtubules are growing, while others are undergoing rapid breakdown. The rate at which microtubules undergo net assembly, or disassembly (depolymerization), varies with mitotic stage [6]. In the present context, depolymerization-based electrostatic attractions are responsible for poleward force generation at kinetochores; electrostatic interactions for poleward force production at centrosomes are treated elsewhere [7].

The electrostatic properties of tubulin have been well-studied [8] [9] [10] [11]. Large-scale computer calculations have determined the dipole moment to be as large as 1800 Debye [9] [12]. Tubulin has a large overall charge of -20 (electron charges) at $\mathrm{pH} 7$, and up to $40 \%$ of the charge resides on C-termini [13]. This large net charge on C-termini is integral to electrostatics-based force production at kinetochores (see below).

\section{Results}

In the context of force generation for chromosome motility at kinetochores, 
Miller et al. [3] state that "... our data argue strongly that the Hecl tail is the critical attachment for deploymerization-coupled movements of chromosomes"; and conclude "... the highest affinity interactions between kinetochores and microtubules are ionic attractions between two unstructured domains." This essentially proposes that bound, oppositely charged distributions are the underlying cause for poleward chromosome motions. As mentioned above, we recently published a force calculation between $\mathrm{Hecl}$ charges, modeled as an experimentally known bound volume positive charge- "unstructured" positive charge-at kinetochores, and experimentally known negative charge at kinetochore microtubule free plus ends that agrees with experimental measurements of the poleward force for chromosome motility [4]. Here we provide a force calculation between positively charged Hec1 tails in kinetochores and negatively charged C-termini at and near microtubule free ends that confirms our previous proposal.

Since the lengths of Hecl tails are much longer than the location volumes of C-termini charge distributions, $\mathrm{Hecl}$ tails will be modeled as very long linear charges, with a linear charge density $\lambda \mathrm{C} / \mathrm{m}$ (Coulombs/meter). A simple application of Gauss's law [14] for an infinitely long line charge distribution gives the electric field magnitude at a distance $r$ from the line charge as

$$
E=\lambda / 2 \pi \varepsilon r
$$

where $\mathcal{E}\left(=k \varepsilon_{0}\right)$ is the kinetochore permittivity, $\varepsilon_{0}=8.85 \mathrm{pF} / \mathrm{m}$ (picoFarads/meter), and $k$ is the kinetochore dielectric constant. Note that the relatively small contributions from edge effects near the ends of the Hecl tails are neglected in this calculation.

The N-terminal tail of Hecl contains an equivalent positive charge $Q$ of 10 (electron charges, e) [3], distributed over a distance 1 of $55 \mathrm{~nm}$ [15], giving a linear charge density $\lambda=Q / 1$ of 10 e/55 $\mathrm{nm}=29 \mathrm{pC} / \mathrm{m}$ (picoCoulombs per meter). The force generating interaction is between positively charged $\mathrm{Hecl}$ tails and negatively charged C-termini on concave sides of splaying protofilaments.

For the force per protofilament, we have:

$$
F_{p f}=q E=n e \lambda / 2 \pi \varepsilon r
$$

where $q=n e$ is the charge of $n$ electrons on C-termini of a protofilament interacting with a Hecl tail. Consistent with their open structures, a cytosol-saturated kinetochore is expected to have a dielectric constant midway between the kinetochore $d r y$ value and cytoplasmic water [16]. Since most condensed-matter (dry) dielectric constants are between 1 and 5, the value for cytoplasmic water dominates, and a conservative midpoint value $k=45((80+10) / 2)$ will be assumed [4]. Substituting this value in (2), with $\lambda=29 \mathrm{pC} / \mathrm{m}$, and the distance of the effective charge centers of C-termini charges, $r=3 \mathrm{~nm}$, we have $F_{p f}=0.6 n \mathrm{pN} / \mathrm{pf}$ (picoNewtons per protofilament).

Kinetochores generally number at least $8 \mathrm{Hecl}$ proteins per microtubule [17], and there are 13 protofilaments per microtubule. It will therefore be conserva- 
tively assumed that four protofilaments in a microtubule are interacting with a Hecl tail at any given moment. These subsets would be constantly changing among the microtubules penetrating a kinetochore. Thus, the total force per microtubule $F=4(0.62) n=2.5 n \mathrm{pN} / \mathrm{MT}$ (picoNewtons per microtubule). Equating this to the experimental range $1-5 \mathrm{pN} / \mathrm{MT}$ [18], we find that $n=0.4-2.5$ electron charges. This result, like that of the previous calculation [4], falls well within the observed experimental range [9] [13] [19], and the agreement represents a successful ab initio theoretical derivation of this force magnitude.

Since microtubule C-termini are on the concave sides of progressively splaying microtubules, increasing protofilament curvature will lead to a separation of the charges on Hec1 tails and C-termini. Subsets of low curvature splaying protofilaments produce poleward force, while other subsets of protofilaments with more pronounced curvature in later stages of depolymerization fail to bind. Accordingly, poleward forces are generated as microtubules depolymerize, in agreement with observation.

\section{Discussion}

Electrostatic fields within the cytosol are subject to strong attenuation due to screening by oppositely charged ions (counterion screening), decreasing exponentially to much smaller values over a distance of several Debye lengths. The Debye length within cells is typically given to be of order $1 \mathrm{~nm}$ [20], and since cells have much larger dimensions, one is tempted to conclude that electrostatic force could not be a major factor in providing the cause for chromosome motility in biological cells. However the presence of microtubules challenges that notion. Microtubules can be thought of as intermediaries that extend the reach of the electrostatic interaction over cellular distances, making the second most powerful force in nature available to cells in spite of their ionic nature.

Cellular electrostatics is also strongly influenced by reduced counterion screening due to layered water adhering to charged molecules. Such water layering - with consequent reduction or elimination of Debye screening - at charged proteins has long been theorized [21] [22], and has been confirmed by experiment [23]. Additionally, water between sufficiently close (up to $3 \mathrm{~nm}$ ) charged proteins has a dielectric permittivity that is considerably reduced from the bulk value far from charged surfaces [24] [25] [26]. The combination of these effects (or conditions)-water layering and reduced dielectric constant-can significantly influence cellular electrostatics in a number of important ways. This is especially true in relation to mitosis [26].

\section{Conclusion}

Given positive charge at kinetochores and negative charge on plus ends of microtubules, it is difficult to conceptualize there not being an attractive electrostatic poleward-directed force between these structures. A direct calculation of the electrostatic force between positively charged Hecl tails and negatively 
charged C-termini at and near the free ends of microtubules supports an electrostatic force generating mechanism for poleward chromosome motions during mitosis. A singular strength of the present calculation is that the disassembly rate of microtubules at kinetochores is explicitly shown to be correlated with force production at kinetochores. In a broader context, understanding the underlying forces and mechanisms that dictate chromosome movements through mitosis will be critical to the development of approaches to circumvent anomalous cell divisions (e.g., cancer).

\section{Authors' Contributions}

LJG conceptualized the theoretical aspects of this article and DHS provided intellectual contributions. Both authors read and approved the final manuscript.

\section{Conflicts of Interest}

The authors declare they have no competing interests.

\section{References}

[1] Gagliardi, L.J. (2002) Electrostatic Force in Prometaphase, Metaphase, and Anaphase-A Chromosome Motions. Physical Review E, 66, Article ID: 011901. https://doi.org/10.1103/PhysRevE.66.011901

[2] Gagliardi, L.J. (2005) Electrostatic Force Generation in Chromosome Motions during Mitosis. Journal of Electrostatics, 63, 309-327. https://doi.org/10.1016/j.elstat.2004.09.007

[3] Miller, S.A., Johnson, M.L. and Stukenberg, P.T. (2008) Kinetochore Attachments Require an Interaction between Unstructured Tails on Microtubules and Ndc80/Hec1. Current Biology, 18, 1785-1791. https://doi.org/10.1016/j.cub.2008.11.007

[4] Gagliardi, L.J. and Shain, D.H. (2016) Electrostatic Forces Drive Poleward Chromosome Motions at Kinetochores. Cell Division, 11, 14. https://doi.org/10.1186/s13008-016-0026-1

[5] Nicklas, R.B. and Kubai, D.F. (1985) Microtubules, Chromosome Movement, and Reorientation after Chromosomes Are Detached from the Spindle by Micromanipulation. Chromosoma, 92, 313-324. https://doi.org/10.1007/BF00329815

[6] Alberts, B., et al. (1994) Molecular Biology of the Cell. 3rd Edition, Garland, New York, 920.

[7] Gagliardi, L.J. and Shain, D.H. (2014) Polar Electrostatic Forces Drive Poleward Chromosome Motions. Cell Division, 9, 5. https://doi.org/10.1186/s13008-014-0005-3

[8] Sataric, M.V., Tuszynski, J.A. and Zakula, R.B. (1993) Kinklike Excitations as an Energy Transfer Mechanism. Physical Review E, 48, 589-597. https://doi.org/10.1103/PhysRevE.48.589

[9] Brown, J.A. and Tuszynski, J.A. (1997) Dipole Interactions in Axonal Microtubules as a Mechanism of Signal Propagation. Physical Review E, 56, 5834-5840. https://doi.org/10.1103/PhysRevE.56.5834

[10] Baker, N.A., et al. (2001) Electrostatics of Nanosystems: Applications to Microtubules and the Ribosome. Proceedings of the National Academy of Sciences, 98, 10037-10041. https://doi.org/10.1073/pnas.181342398 
[11] Tuszynski, J.A., Brown, J.A. and Hawrylak, P. (1998) Dielectric Polarization, Electrical Conduction, Information Processing and Quantum Computation in Microtubules: Are They Plausible? Philosophical Transactions of the Royal Society of London. Series A, 356, 1897-1926. https://doi.org/10.1098/rsta.1998.0255

[12] Tuszynski, J.A., et al. (1995) Ferroelectric Behavior in Microtubule Dipole Lattices: Implications for Information Processing, Signaling and Assembly/Disassembly. Journal of Theoretical Biology, 174, 371-380. https://doi.org/10.1006/jtbi.1995.0105

[13] Tuszynski, J.A., et al. (2002) Electrostatic Properties of Tubulin and Microtubules. In: Crowley, J.M., Zaretsky, M. and Kazkaz, G., Eds., Proceedings of the Electrostatics Society of America and Institute of Electrostatics-Japan, Laplacian Press, Morgan Hill, 41-50.

[14] Halliday, D. and Resnick, R. (1962) Physics. Vol. 2, John Wiley \& Sons, New York, 698.

[15] Wilson-Kubalek, E.M., et al. (2008) Orientation and Structure of the Ndc80 Complex on the Microtubule Lattice. The Journal of Cell Biology, 182, 1055-1061. https://doi.org/10.1083/jcb.200804170

[16] Schelkunoff, S.A. (1963) Electromagnetic Fields. Blaisdell, New York, 29.

[17] Joglekar, A.P., et al. (2006) Molecular Architecture of a Kinetochore-Microtubule Attachment Site. Nature Cell Biology, 8, 581-598. https://doi.org/10.1038/ncb1414

[18] Grishchuk, E.L., et al. (2005) Force Production by Disassembling Microtubules. Nature, 438, 384-388. https://doi.org/10.1038/nature04132

[19] Stracke, R., et al. (2002) Analysis of the Migration Behavior of Single Microtubules in Electric Fields. Biochemical and Biophysical Research Communications, 293, 602-609. https://doi.org/10.1016/S0006-291X(02)00251-6

[20] Benedek, G.B. and Villars, F.M.H. (2000) Physics: With Illustrative Examples from Medicine and Biology: Electricity and Magnetism. Springer-Verlag, New York, 403. https://doi.org/10.1007/978-1-4612-1240-9

[21] Jordan-Lloyd, D. and Shore, A. (1938) The Chemistry of Proteins. JA Churchill Publishing Company, London. https://doi.org/10.1021/ja01220a017

[22] Pauling, L. (1945) The Adsorption of Water by Proteins. Journal of the American Chemical Society, 67, 555-557.

[23] Toney, M.F., et al. (1994) Voltage-Dependent Ordering of Water Molecules at an Electrode-Electrolyte Interface. Nature, 368, 444-446. https://doi.org/10.1038/368444a 0

[24] Bockris, J.O. and Reddy, A.K.N. (1977) Modern Electrochemistry. Plenum, New York.

[25] Teschke, O., Ceotto, G. and De Souza, E.F. (2001) Interfacial Water Dielectric Permittivity-Profile Measurements Using Atomic Force Spectroscopy. Physical Review E, 64, Article ID: 011605. https://doi.org/10.1103/PhysRevE.64.011605

[26] Gagliardi, L.J. (2009) Electrostatic Considerations in Mitosis. iUniverse Publishers, Bloomington, IN. 